\title{
Las FAMIPYMES como un modelo de desarrollo en la economía de Tungurahua.
}

\section{The FAMIPYMES as a model of development in the economy Tungurahua.}

Hugo Orlando Bonilla Vásconez. ${ }^{1}$, Adela Maritza Moncayo Robinson. ${ }^{2}$ \& Lourdes Elizabeth Solís Freire. ${ }^{3}$

\section{DOI: https://doi.org/10.33262/visionariodigital.v1i1.232}

\section{Resumen.}

"El presente artículo trata sobre la "Las Famipymes en la economía", las famipymes son organizaciones en las cuales la toma de decisiones está influenciada por los miembros de una familia, por ende el crecimiento o la decadencia de la empresa depende de la capacidad para controlar que tiene cada uno de los familiares, debido a que los integrantes de la compañía son miembros de una familia. Las famipymes en la economía son muy importantes debido a que empiezan como emprendimientos familiares y con el pasar del tiempo se van desarrollan y por ende se posesionan en un mercado meta, lo cual les permite mejorar su economía familiar y a la vez mejorar la economía del país ya que son entidades que ayuden a contribuir al estado. Además, están empresas aparecen por los inicios o ideas de un pilar fundamental de la familia como puede ser un padre o una madre, los cuales en un futuro desean que sus negocios estén a cargo de sus hijos y así puedan crecer y desarrollar nuevas ideas. En el cual abordaremos los siguientes temas definición, características, importancia, ventajas, planificación, fortalezas y debilidades de las famipymes.

Palabras clave: Empresas familiares, Pymes, Economía, Microempresa, Servicios, Precios.

\begin{abstract}
.
"This article is about" The Famipymes in the economy, "the famipymes are organizations in which decision making is influenced by family members, hence the growth or decline of the company depends on the ability to check that has each of the family, because the members of the company are members of a family. The famipymes in the economy are
\end{abstract}

${ }^{1}$ Universidad Técnica de Ambato, FCADM, Ecuador, hugobv_1991@hotmail.com

${ }^{2}$ Universidad Técnica de Ambato, FCADM, Ecuador, adelita_leli24@hotmail.com ${ }^{3}$ Universidad Técnica de Ambato.FCADM.Ecuador,elizabethlourdes17@gmail.com 
very important because they start out as family enterprises and over time they will develop and thus take possession in a target market, enabling them to improve their family finances while improving the economy as they are entities that help contribute to the state. Then there are companies appear early or ideas of a fundamental pillar of the family such as a father or a mother, who in the future want their businesses to be in charge of their children so they can grow and develop new ideas. In which we will address the following topics definition, characteristics, importance, benefits, planning, strengths and weaknesses of the famipymes.

Keywords: Family businesses, SMEs, Economics, Micro, services, prices.

\section{Introducción.}

El arte de combinar empresa y familia conlleva a enfrentar día a día dos conceptos que, en apariencia, son antagónicos: el Amor y el Dinero. Pero no hay nada más lejos de la realidad. El manejo de dos mundos tan disímiles y complejos al mismo tiempo, la empresa y la familia, convierten las estrategias de gestión en un verdadero arte que mezcla creatividad, experiencia y visión para responder al compromiso que implica el compartir el trabajo con los seres amados.

En el manejo académico del tema, lo novedoso reside en el haber encontrado algunas claves para llevar armónicamente la confrontación de las necesidades de la familia y el negocio, buscando métodos especializados que logren mantener el funcionamiento del sistema a través de una familia unida, una empresa sana, la preservación del patrimonio y la continuidad generacional. A lo largo de estos artículos iremos descubriendo los principales conceptos alrededor de la Empresa Familiar, sus características, modelos explicativos y las problemáticas más comunes, discutidas en ocasiones a través de las típicas preguntas a un consultor de Empresa Familiar.

El principal problema con este tipo de compañías es que suelen dar por sentado que las relaciones familiares son las que deben dominar en la empresa. Es decir, trasladan a la formalidad de una organización profesional la informalidad del trato familiar. Con el tiempo, el crecimiento del sistema familia-empresa-propiedad exigirá un tratamiento más profesionalizado, donde será necesaria la correcta aplicación de las reglas de un juego tan complicado. Una Empresa Familiar ideal sería aquella que le garantiza a cada uno de sus miembros el escenario óptimo para su desarrollo integral, tanto en el aspecto económico como en el ético y el afectivo.

En el fondo, toda empresa que desee preservar su carácter familiar, debe tener la capacidad de profesionalizarse (esto incluye atraer y retener a directivos no familiares), y planificar la sucesión de su dirección (incluyendo el saber ceder la batuta en su momento y garantizar la competencia del sucesor). (Salazar, 2005)

La investigación en la empresa familiar ha conseguido en los últimos años una proyección que puede ser apreciada en el volumen de trabajos publicados en revistas especializadas como no especializadas. Su estudio ha traspasado las fronteras desde investigaciones que 
evolucionan con trabajos descriptivos, pasando por aquellos que se limitan a utilizar a la empresa familiar como un ámbito de aplicación, hasta los que desarrollan interpretaciones propias del fenómeno para dar respuesta a la problemática del campo.

Desde finales del siglo pasado, los estudiosos están intentando reflejar un conocimiento más amplio que se plasma en los aspectos intrínsecos y extrínsecos de la investigación. Entendido el conocimiento de un ámbito de estudio como un proceso acumulativo del aprendizaje colectivo, el presente artículo intenta abordar una reflexión sobre los elementos característicos que potencialmente se le atribuyen a un campo en creación y sobre los cuales se reflejan los parámetros de independencia.

La empresa y la familia se articulan en una institución social productiva que tiene su origen en la evolución histórica de las sociedades. En el ecuador de la edad media comienza a notarse la configuración de un incipiente sistema de mercado amparado por los propios estados entre oriente (Península Ibérica y el Magreb) y occidente (China). Sin embargo, a partir del siglo XVI se produce la transformación de un sistema basado en la tierra (marcadamente medieval) a otro capitalista basado en el dinero, las finanzas y la banca, para convertirse, con la primera revolución industrial, en un capitalismo industrial. Valdalizo y López García (2000) destacan que entre los siglos XVI y XVIII, antes de que surgieran los sistemas legales que garantizasen los contratos, los comerciantes y artesanos desarrollaron códigos de conducta cuando existía una cohesión social fuerte que impulsaba el comercio. Así surge la importancia de las relaciones familiares en los negocios como motor aglutinante del sistema económico que permitía el intercambio ante la incertidumbre de un marco que lo legislase. (Basco, 2006)

En la actualidad la manera que los empresarios ven el modo de seguir y transcurrir en la vida se ha dado la forma que surjan más empresas familiares que ayuden con la economía de cada una de las familias. Se puede solo observar en el Ecuador que personas ven la necesidad de seguir incursionando en su vida y a su vez dar fuentes de trabajo a todas las personas que estén desempleadas.

La virtud en sí que genera el crear más empresas (Pymes) son de gran ayuda para el país a si puede ayudar a crecer más con los emprendimientos de cada una de las personas de acuerdo a la necesidad que tenga.

Como estamos pasando una situación importante en el ecuador nos hemos enfocado a tratar este tema ya que hemos visto que hoy en día la mayoría de empresas que han crecido son las micro-empresas y pequeñas empresas, se podría decir que es una ayuda de seguir con los esfuerzos encaminados de cada familia de salir adelante, por el motivo que se ve más grave la situación de convivencia cada día la vida es más dura y sobre todo los precios de los productos suben y bajan, es decir no tenemos una economía estable que favorezca a la sociedad de manera que cada uno de ellos se ve la manera de salir adelante y sobrevivir en cualquier etapa de la vida.

Las famipymes son aquellas que contribuyen a la economía de las familias y aquellas que ayudan a que productos que no habían salido a la exposición sean de más agrado para la sociedad. 
Las empresas familiares son muy importantes en la economía de cualquier país ya que estas forman parte del desarrollo económico del mismo, sin embrago este tipo de empresas también enfrentan varios problemas y el más importante es el que las estadísticas demuestran una alta tasa de mortalidad de las mismas debido a varios factores como los conflictos familiares y estos se trasladan a la empresa, también es muy común Discrepancias remunerativas entre empleados familiares y no familiares. Además de surgir otros problemas como lo es el que los familiares ocupen puestos directores sin la preparación adecuada, lo que genera problemas con directivos externos. Un problema común también es el estilo de dirección autocrático que manejan estas empresas, se centra el poder en una sola persona por lo que es un punto débil en estas empresas familiares.

\section{Problema.}

Determinar qué porcentaje de las empresas existentes son empresas familiares y cuáles son sus ventajas al ser consideradas familiares.

\section{Revisión de literatura.}

Los desafíos que enfrentan las empresas familiares suelen estar vinculados al modo de resolver los problemas que se presentan entre los miembros de la familia y al grado de aceptación de los niveles superiores de delegación en los delegados profesionales. Existe un proceso de renovación de la gestión que pasa inevitablemente por conceder cada vez más competencias a gestores mejor preparados para atender las distintas áreas de la empresa. Una fórmula que se ha practicado para regular las relaciones entre la empresa y los integrantes de una familia es la aprobación de un "protocolo familiar", es decir una suerte de carta de navegación para la gestión y prevención de los conflictos parentales. Es un instrumento que sólo vincula a los miembros de la familia y es independiente del estatuto societario. En algunos casos se ha concebido la presencia de un "consejo de familia" para que actúe como autoridad arbitral para mediar en los conflictos y evitar así que se trasladen a la empresa. Se pueden establecer normas de comportamiento, incentivos y sanciones para evitar cualquier conflicto familiar dentro y fuera de la organización, debido a que esto afecta la economía de la entidad y podría incluso obtener una pérdida lo cual no es recomendable en una empresa. Las famipymes corren un riesgo grande el cual es deteriorar la relación familiar, por ello es importante realizar cada una de las actividades de la organización de manera adecuada, para evitar todo tipo de conflictos. La finalidad de una compañía es mejorar la rentabilidad, mientras que el parámetro de éxito en una familia es conservar la armonía entre sus miembros. Otros de los factores afectados de estas empresas consisten en la resistencia del fundador o propietario a asumir mayores niveles de profesionalización de la gestión. Un emprendedor originario de estas entidades ha tenido éxito el cual se convierte en un resultado de percepción que le permite conocer un nicho de mercado en el cual va a operar y a la vez a obtener clientes los cuales le permiten crecer en cuanto a su productividad y a ingresos económicos, esto será el resultado de la suma de esfuerzos de los miembros de 
la organización y a la vez le permite superar las barreras de ingresos. En estas empresas prevalece la confianza por encima de los controles, en vista de ser una entidad familiar. En este tipo de empresas tanto los integrantes de la familia como los empleados comparten una cultura, como resultado de compartir mucho más que un ambiente laboral, la cultura familiar se traslada a la empresa y es fácilmente asimilable por los empleados, obviamente esto será beneficioso en la medida que esta cultura se introduzca dentro de las estrategias de la empresa, las facilite y no las dificulte.

\section{Metodología.}

La Investigación Según (Tamayo, 2004) es un proceso que, mediante la aplicación del método científico, encamina a conseguir información apreciable y relevante para concebir, comprobar, corregir o emplear el conocimiento de procedimientos utilizados para alcanzar los objetivos. Para desarrollar este proyecto se utilizó la investigación bibliografía - documental debido a que se basó en libros, informes técnicos de los diferentes autores para respaldar la investigación realizada.

Se recurre a la investigación de campo, debido a que esta nos permite conocer el problema que existe en las instituciones educativas como es la falta de superación de los docentes en la multimedia.

(Flores, 2013) El primer punto para que una famipyme siga activa en el mundo globalizado, es definir un modelo de actuación empresarial donde se pone en manifiesto el distanciamiento de la relación familiar con lo laboral para mitigar los posibles conflictos que se ocasionan dentro de las entidades y por los cuales hay que tomar las mejores decisiones.

En este tipo de empresas la sucesión es entendida como "la continuación del legado", pero que lastimosamente no ha llegado a ser exitoso en mucho de los casos, en vista de que las empresas no cambian la forma de gestionar cada una de las actividades,

La sucesión puede ser determinante para la empresa familiar ya que al producirse una transición de poder la empresa ya no volverá a ser la misma, por lo que es necesario que los actuales decisores sepan evaluar si la decisión de ceder su lugar deba recaer por consanguineidad o por capacidad empresarial, esto en el caso de ver a la empresa en un futuro como una corporación.

En cualquiera de los casos, los conocimientos, experiencia, pensamiento estratégico serán las bases para que la entidad obtenga la renovación solicitada por los cambios del medio. Algunos expertos recomiendan que el cambio de mando debe manejarse como algo natural y que en caso de tener por sucesión sanguínea se prevea un aislamiento del sucesor, esto quiere decir que deberá vincularlo a la actividad empresarial desde "extremo a extremo", es decir que adquiera experiencia y pueda definir el área donde tenga mejor rendimiento y que estará asociado con la formación académica.

En el caso de la sucesión a un tercero es oportuno apoyarse en modelos de gobierno corporativo para tener monitoreo y control del desarrollo empresarial y que esta de la mano con la intensión de crecimiento continuo. 
Una vez revisado el tipo de sucesión el siguiente paso es la planificación estratégica, en la cual toda empresa sin importar su tamaño tiene que afianzar su direccionamiento estratégico, mismo que consiste en definir a donde se quiere llegar a través del tiempo (visión), la razón de ser (misión), los valores y los objetivos del negocio.

Definiendo el rumbo del negocio se diseñarán los planes de acción, de manera que se fortalezcan los factores básicos de competencia de la empresa sin descuidar el esquema de seguimiento y evaluación que se puede plasmar en un Balance Scorecard.

El BSC conocido como balance scorecard facilita a las empresas familiares la integración del día a día (corto plazo) con los planes futuros (largo plazo), y lo prepara para afrontar de mejor manera el reto de ser rentables y crecer.

\section{Estado del arte.}

Según (Montoya, 2012) dice: El estado del arte es una modalidad de la investigación documental que permite el estudio del conocimiento acumulado (escrito en textos) dentro de un área específica. Sus orígenes se remontan a los años ochenta, época en la que se utilizaba como herramienta para compilar y sistematizar información especialmente el área de ciencias sociales, sin embargo, en la medida en que estos estudios se realizaron con el fin de hacer balances sobre las tendencias de investigación y como punto de partida para la toma de decisiones, el estado del arte se posicionó como una modalidad de investigación de la investigación. Hoy en día se considera que en general, el estado del arte puede abordarse desde tres perspectivas fundamentales. Sea cual fuere el abordaje del estado del arte, se considera que su realización implica el desarrollo de una metodología resumida en tres grandes pasos: contextualización, clasificación y categorización; los cuales son complementados por una fase adicional que permita asociar al estado del arte de manera estructural, es decir, hacer el análisis (sinónimo de investigación).

De esta manera se observa que la realización de estados del arte permite la circulación de la información, genera una demanda de conocimiento y establece comparaciones con otros conocimientos paralelos a este, ofreciendo diferentes posibilidades de comprensión del problema tratado; pues brinda más de una alternativa de estudio.

1. Contextualización. - Las famipymes en la economía en la actualidad son importantes por la flexibilidad de adaptarse a los cambios tecnológicos y por su gran potencial de generar empleos. Además representan un medio para impulsar del desarrollo económico y una mejor distribucion de la riqueza tanto del país como de una familia.

\section{Categorización:}

1. Las famipymes en la economía.

1.1. Definición de las famipymes

1.2. Características de las famipymes

1.3. Importancia de las famipymes

1.4. Ventajas de las famipymes 
1.5. Planificación de las famipymes

1.6. Fortalezas y debilidades de las famipymes.

\section{Discusión.}

Las Empresas familiares, son las unidades económicas más antiguas del mundo. Inicialmente, fueron producciones que autoabastecían a la familia; luego extendieron su actividad por medio del trueque, y se insertaron finalmente en el ciclo económico como se las conoce en la actualmente. Estas empresas con el pasar del tiempo han crecido gracias a los esfuerzos que realizan los miembros de la familia, además se han incrementado por la habilidad de las personas para innovar o crear nuevos productos y ofrecer a un nicho de mercado para de esta manera mejorar la situación económica de la familia y a la vez satisfacer las necesidades de la comunidad. Se puede decir que estas empresas han surgido porque el propietario ha decidido ser una persona independiente y obtener mejores ingresos. Las famipymes son entidades que brindan mayor oportunidad de empleo a la sociedad para que puedan sobrevivir. La empresa familiar representa el esfuerzo acumulado de sus miembros durante muchos años. Este incluye decisiones aún a costa del sacrificio de beneficios personales, dando como resultado la constitución de un patrimonio mediante la unión de la entidad económica que hoy se encuentra en plena operación.

En las empresas familiares los múltiples inconvenientes surgen porque los roles de dueño, directivo y familiar no son claramente delimitados y las acciones que conciernen a cada rol suelen ser confundidos. Ahora se aborda sobre los retos más comunes que las empresas familiares enfrentan como: comunicación, equidad, responsabilidad y planeación de la sucesión-continuidad. La comunicación es fundamental en todos los aspectos del ser humano. En las empresas familiares es transcendental porque es más complejo abordar temas dolorosos, controversiales o difíciles. Para ello, los temas álgidos deben ser tratados entre todos los implicados y moderado por el miembro familiar más conciliador. En el caso que haya intereses entre todos los miembros familiares es conveniente contar con un actor externo que guíe la resolución de conflictos. La equidad también se ve afectada por los diferentes roles en el negocio que ocupa cada familiar. Debido a las habilidades particulares de cada persona, preparación, contribuciones, oportunidades visualizadas e incluso frente a los miembros que no participan del todo en el negocio; se introduce un elemento de inequidad desde la perspectiva del deseo familiar de justicia equitativa. Esta situación, aunque lo persigue la familia, es algo que difícilmente ocurre porque, por ejemplo, no todos pueden ser gerente general y percibir igual sueldo cuando existen diferentes responsabilidades. Por tanto, los cargos y remuneraciones tienen que basarse en funciones y resultados. Tomando el aspecto de resultados, el reto que está íntimamente ligado con este concepto es el de la responsabilidad. En otras palabras, cada miembro familiar es responsable de sus resultados, aunque a veces resulte incómodo un reclamo a hijos, nietos o esposa incluso enfocándolo estrictamente en el área de negocio. Es normal que los miembros familiares encuentren cierta dificultad para cuestionar a otros e 
inclusive dar paso a un proceso de evaluación. Sin embargo, por el bien de la empresa estos aspectos deben llevarse a cabo bajo el amor, aceptación y apoyo familiar debido a que la rentabilidad está en función de resultados óptimos. El último reto es la sucesión. Así como la familia es un espacio para producir adultos que formarán la próxima generación, los negocios tienen la misma necesidad. Usualmente los miembros de edad avanzada encuentran difícil salir ordenadamente y transferir la estafeta a la generación nueva. Aquí la premisa es que las generaciones que vienen estén preparadas y con más herramientas para impulsar el negocio antes de que la generación que le precede esté dispuesta a retirarse. Todo negocio necesita revitalizarse y una fuente de innovación se encuentra en la descendencia que ha tenido la oportunidad de prepararse de mejor manera.

\section{Resultados.}

\section{Definición de las Famipymes.}

Según (Regalado , 2006), (Cleri , 2007), (Urbano \& Toledano , 2008), (Casani, Llorente, \& Pérez, 2009) Las pymes cuentan con la mas amplia gama de giros productivos, comercializadores y de servicios y tienen la tendencia a relaizar actividades autónomas que tienen relacion con las empresas mas grandes, y de este modo sufriendo la influencia de estas que suelen someterlas, minimizando sus posibilidades de desarrollo o establecen una interdependencia.Las Pymes se vuelven sistemas creadores de valor económico cuando desarrollan dinámicas productivas y competitivas que les permiten enriquecer su contexto conectando a las personas con los mercados, en los cuales se encuentran los recursos requeridos para satisfacer sus necesidades, a la vez que se enriquecen en el proceso. La Pyme es toda aquella empresa que ocupa a menos de 250 personas, cuyo volumen de negocios anual no excede de 50 millones de euros o cuyo ballance general no supera los 43 millones de euros. La Pymes es un colectivo bastante heterogeneo, a su vez que hayan sido clasificadas en microempresas, pequeñas y empresas medianas. Las pequeñas y medianas empresas ( las pymes) desempeñan un papel decisivo en el crecimiento de la economia española y en la creacion de puestos de trabajo. Son un facor clave de estabilidad, y su capacidad para adaptarsed a los ciclos económicos las convierte en una unidad económica imprescindible.

Las famipymes son aquellas que contribuyen en la situacion de las familias y que pueden dar una facilidad mas relevante en la vida de cada uno de ellos, esto contribuye que cada familia ayude a crear mas fuentes de trabajo y que sea mas factible para que el desempleo no se refleje mucho y sobre todo permita aumentar la posibilidad de que crezcan en su pais de origen. 
Las famipymes son aquellas que involucran a las personas y familias sobre como pueden ayudar a la sociedad de manera que contribuyan a satisfacer las necesiades de las personas.

Las pymes son empresas que elaboran las familias por el bien de cada uno de los miembros y por su economia para que se pued aestablecer y sobre todo que puedan pasar las recesiones que tiene cada pais o a su vzs que se devalue la moneda de ese mismo. Cada familia a tenido la necesidad de invertir para una empresa, la cual sea de mas ayuda para la economía y sobre todo que fomente la menra mas explicativa de hacer las cosas en dicha empresa sin dejar de lado las cosas juridicas de cada empresa, contribuyendo a que los productos de emprendimiento sea de ayuda para el pais o para la economia de cada uno.

\section{Características de las Famipymes.}

$>$ (Ronqullo, 2006), (Mucc, 2008), (Urbano \& Toledano , 2008), (Casani, Llorente, \& Pérez, 2009) Las empresas familiares difieren en una serie de aspectos importantes con respecto a las empresas institucionales. Estas tienen que ser capaces de evaluar las dinámicas que rigen las conductas de su fundador, la familia y la organización en su conjunto.Necesitan desarrollar habilidades especiales que les permitan identificar y resolver los problemas que plantean estas dinámicas y adoptar estrategias ingeniosas para fomentar el crecimiento de la empresa, transferir el poder y el control de esta.Poder encontrar empresas familiares en casi todos los sectores, pero en donde han probado tener éxito y una presencia relevante es en el sector comercial y de servicios, donde de la administración está controlada por su propietario-fundador y familiares más cercanos, donde la atención personalizada es fundamental para dar satisfacción a los requerimientos de los clientes. Mantienen un marcado estilo autocrático que las hace resistentes a los cambios., Muchos miembros de la familia, ocupan cargos directivos., Se aprecia una excesiva rigidez en el liderato, lo que impide el desarrollo de otras personas para acceder a puestos claves.,. La expresión aparece como un valor casi irreemplazable y motivo de escasa discusión., No se admite el cuestionamiento del poder., Se valora mucho más la fidelidad que la habilidad. Existen dificultades o se trata la incorporación de expertos no familiares., Son, por lo general, pequeñas y medianas empresas, en sus inicios., Tienen a familiarizar todas las relaciones, incluyendo las que existen con proveedores y clientes., Los antepasados surgen, en ocasiones, como mitos intocables y altamente idealizados., La superación directiva, deviene de la posición familiar., La elección del futuro, por parte de los jóvenes está en relación directa con las expectativas de la familia-empresa., Existe una marcada identificación de los valores organizacionales y familiares., Los conductores, por una parte, pretenden imponer la continuidad incorporando a los jóvenes pero, por otra, rechazan sus ideas o sugerencias., Existen un alto compromiso con algún producto o servicio 
que forma parte de la tradición y que se lo identifica con el "apellido familiar", lo que obstaculiza la eventual desactivación de los mismos y su análisis estratégico. La capacidad de voto mayoritaria debe residir en los miembros de la familia que fundaron la compañía o en los que los sucedieron heredando el patrimonio y en muchas ocasiones la gestión de la misma.

Esa mayoría de votos se puede tener de manera directa o indirecta.

Al menos un representante de la familia o pariente debe participar en la gestión o gobierno de la compañía.

A las compañías cotizadas (aquellas cuya propiedad se encuentra fragmentada en acciones y cuyo principal accionista tiene menos del 50\% de los derecho de voto) se les aplica la definición de empresa familiar si la persona que fundó o adquirió la compañía, o sus familiares o descendientes poseen el $25 \%$ de los derechos de voto. Es decir, aunque la familia no cuente con la mayoría de los votos de la empresa, sí debe ejercer, a través de su participación accionarial, una influencia decisiva.

Las relaciones de afecto: Se trata de lazos que perdonan, que salvan una situación, que a la postre buscan el bien recíproco y a él se someten.

Enorme comprensión: Todos saben cómo piensa el otro, casi se oyen pensar. Se precisa muy poca comunicación formal para conocer la opinión del otro.

Aceptación de la autoridad: De la autoridad del padre, o de la madre, o del hermano que le precede a uno.

Finalidad común: La familia es una unidad de convivencia donde cada uno se realiza y se desarrolla; es una unidad básica y profunda.

Para constituir una famipymes el fundador o descendiente debe tener por lo menos un $25 \%$ de los derechos de voto de los miembros de la compañía o la familia, esto con el fin de que dicha persona sea la idónea para dirigir la empresa familiar y asi obtener mayor ingreso para la empresa, estas empresass están constituidas por miembros de una familia, los mismos que deben tener habilidades para poder solucionar los problemas que se presenten en el transcurso del crecimiento de la organización, para lo cual buscarán una gama de estrategias para ser una empresa pionera en el sector comercial o de servicos que son los sectores en los cuales existen mayor número de famipymes y los que permiten que las familias sobrevivan y puedan desarrollar sus ideas e innovar para que en un futuro sean lideradas por sus descendientes o herederos. La atención personalizada que brindan estas empresas es una de las características muy importantes debido a que de esta manera satisfacen las necesidades de los clientes porque lo más importante en una empresa es estar en la mente de sus consumidores porque de ellos depende la rentabilidad de la empresa. Dentro de las famipymes sew valora la fidelidad más no la habilidad,debido a que si los miemnros familiares divulgan la información de la entidad, la empresa o los miembros de la organización no podrán cumplir con sus objetivos planteados y esto ocacionará conflictos familiares y por ende no habrá un progreso de la empresa. El futuro de los jóvnes esta en las empresas familiares puesto que estas han tenido éxito en el sector 
de los servicios y comerciales, por ello es recomendable que los jóvenes tengan ideas de emprender negocios familiares los cuales les permitirán crecer y ser personas pioneras en el mercado. El producto o servico que ofrecebn estas entidades debe tener el apellido familiar para poder tener mayor peso y así darle relevancia a la familia y por ende a la empresa y poder ganar nuevos clientes por tener un producto altamnete identificado y de calidad. Las relaciones de estas organizaciones son recíprocas poruqe buscan el bien tanto familiar como para la empresa. La comprensión es una de las características que siempre debe prevalecer en estas empresas ya que la autoridad precede del padre o de la madre para un subordinado, en caso de que nio exista esto la empresa no podrá funcionar, poruqe una familia es una convivencia donde cada uno de sus miembrois crecen o se desarrollan por medio del impulso del pilar fundamental de la familia.

\section{Importancia de las Famipymes .}

> Según (De Aguilar, 1998), (Regalado , 2006), (Goméz Betancourt, 2006), (Martínez Echezárraga, 2011), Las pequeñas y medianas empresas PYME’s, tienen particular importancia para las economías nacionales, no solo por sus aportaciones a la producción y distribucion de bienes y servicios, si no también por la flexibilidad de adaptarse a los cambios tecnológicos y gran potencial de generacón de empleos. Representan un excelente medio para impulsar del desarrollo económico y una mejor distribucion de la riqueza.

Hoy día, los gobierno de paises en desarrollo reconocen la impportancia de las PyME's por su contribución al creciemiento economico, a la generacion de empleo, asi como al desarrollo regional y local. La importancia de empresa familiar (EF) en la economía de un país es un hecho indiscutible, más aún si son analizadas las consecuencias de sus actividades empresariales en el desarrollo integral de una sociedad.

En una economia de mercado, el empresario es la pieza clave. Si no hay empresarios, no hay empresa, no hay creación de puestos de trabajo ni de riqueza. Dentro del conjunto de empresas, las familiares constituyen la espina dorsal del desarrollo económico. La mayoria del mundo son de propiedad familiar. Es preciso advertir, sin embargo, que muchos estudios no distiguen el concepto de empresa familiar del de empresa personal, es decir, de un solo dueño muchas de las cuales se convierten mas tarde en familiares. Algunos expertos estiman en un tercio la influencia de las empresas personales dentro de las cifras mencionadas. No resulta fácil estimar el peso de la empresa familiar en el seno de la economía de un país, ya que, además de considerar a la mayor parte de las PYMEs, es necesario agregar, como se ha comentado, algunas grandes empresas, que, no obstante, funcionan con el modelo gerencial de tipo familiar. De lo que no cabe duda es de su enorme importancia, especialmente en lo que se refiere al número de personas empleadas. 
La importancia de las famipymes contribuyen a las empresas de manera que pueden ayudar a la empresa a incrementar mas con su economía y sobre todo que sepamos las maneras de como ir creciendo en el campo empresarial de manera que se fomente a las personas el impulso de crecer como empresa e impulsar dia a dia la rentabilidad de la empresa. Las pymes son pequeñas empresas que han ido ocupando mas terreno en el mundo y a su vez contibuyendo con la situacion de los paises.

Esto contribuye a que todas las empresas pequeñas ayuden a la satisfacción de las necesidades de las personas o tambien a las de un pais, teniendo en cuenta las ventajas y desventajas que pueden tener las empresas pequeñas ya que es un mundo transcendiente que no es facil el poner un empresa deben tener en cuenta algunos factores que involucren a la empresa y a su alrededor.

\section{Ventajas de las Famipymes.}

Según (Ronqullo, 2006), (Hambra, 2013), (Ruiz, 2011), (Bueno, 2014), Las ventajas de estas entidad son.- Compromiso, conocimiento, flexibilidad en el trabajo, tiempo y dinero, planeación a largo plazo, cultura familiar estable, rapidez en la toma de decisiones, confiabilidad y orgullo. El sentido de lucha y la capacidad de resiliencia. Es decir, la capacidad no sólo de soportar los momentos difíciles juntos, sino de lograr que ello los haga más fuertes y exitosos. Siendo gran parte fundadas por inmigrantes, o sus descendientes, las familias empresarias argentinas están marcadas por una épica de la privación y por un fuerte deseo de superarla. Es así que muchas de ellas son austeras aún en la riqueza (y generalmente son las más exitosas).Hay un fuerte sentido del éxito, ligado a la capacidad de trabajo y a la aplicación de la inteligencia y el esfuerzo por lograr metas exigentes. El sentido de pertenencia y la capacidad de resistir la adversidad están ligados a una forma de proyecto trascendente que no tienen los ejecutivos de las empresas anónimas. Entre las principales ventajas se puede definir como básica, un mayor nivel de compromiso de los integrantes debido a sus lazos familiares Cuentan con valores claros. -Es común ver como los valores de la familia se trasladan a la empresa familiar. Ello constituye una de sus principales fortalezas, su identidad única. La dirección tiene autoridad total para tomar decisiones. - Las desventajas "burocráticas" de las empresas trasnacionales o de los grandes corporativos se dan en razón de su diversidad de socios y/o a la sofisticación de su estructura de organización. Una empresa familiar es mucho más ágil y su director tiene gran libertad; con ello, adquiere superioridad frente a las que no lo son. Hay una clara tendencia a pensar en el largo plazo. -En consecuencia de que el gran interés de los fundadores y aún de los sucesores es el bienestar de la familia, las decisiones se toman más pensando en el futuro que en la conveniencia inmediata o del corto plazo. Para la dirección es importante un ambiente de armonía. Derivado de que la filosofía de la familia empresaria se traslada a la empresa, la armonía que se procura en la familia, se busca también en la organización, incluyendo al personal, clientes y proveedores. 
Las ventajas que presentan están empresas son el conocimiento, flexibilidad en el trabajo es decir los miembros de la compañía deben adoptarse a los cambios debido a que no solo son parte de una familia sino también parte de una empresa por ende las actividades cambiarán constantemente de acuerdo a las necesidades que tengan los clientes y la empresa, el tiempo y dinero es otra de las ventajas en vista de que los representantes dela entidad deben tener dinero para poder desarrollar dicho negocio y por ende tener tiempo para poder establecer la empresa legalmente en un sector estratégico, la planeación a largo plazo, cultura familiar estable, rapidez en la toma de decisiones son puntos a favor de estas empresas los cuales se debe desarrollar con responsabilidad total porque si no se los realiza con la seriedad del caso puede ocasionar conflictos familiares y a la vez la pérdida de la empresa es por ello que se recomienda que en la empresa exista confiabilidad entre los miembros de la entidad. Las personas que deciden ponerse empresas familiares son personas luchadoras que deciden resistir a los cambios que se les presente al momento de crear su empresa los cuales no son fáciles de superar pero si posibles de alcanzarlos y más aún cuando existe capacidad y ánimo para realizarlo porque ellos tienen inteligencia y habilidad para poder salir de donde se encuentran y ser personas emprendedoras, tal vez ser un empresario es afrontar a una serie de adversidades, pero esto no quiere decir que no pueden en un futuro crecer más bien es un punto que ayudará a que una empresa y una familia tenga mejores ingresos. Los valores que existen dentro de la familia se constituirán en los valores que tiene la empresa los cuales son importantes para que cada una de las cosas se realice eficientemente y así poder lograr el éxito esperado. La toma de decisiones depende de la dirección de la empresa, en la cual participan todos los miembros de la familia para así tomar la mejor y desarrollar de mejor manera para evitar cuellos de botella al momento de implementar una decisión. Las mismas que son a largo plazo más no a corto plazo porque es el futuro de la empresa y por ende es la economía de una familia que está en constante movimiento, por ello hay que pensar bien las decisiones que se tomen. La armonía que se procura en la familia también es importante tenerla en la organización en vista de que incluye personal, clientes y proveedores y si no existe esto la empresa dejará de funcionar y podrá traer conflictos familiares y los mismos trasladarse a la empresa.

\section{Planificación de las Famipymes.}

> Según (Alexandra, 2013), (Ward, 2003), (Frederick, 2011), (Mucci, 2008), (Echezárraga, 2010), Una empresa familiar está controlada por los miembros de una misma familia en la que el capital y, en su caso, la gestión o el gobierno están en manos de una familia(y personas relacionadas personalmente con dicha familia), que tienen la capacidad de ejercer sobre ella una influencia suficiente para controlarla, y cuya visión estratégica incluye el propósito de darle continuidad en manos de la siguiente generación familiar 
El primer paso se recomienda para que una pyme familiar siga activa en el mundo globalizado, es definir un modelo de actuación empresarial, donde se pone en manifiesto el distanciamiento de relación familiar con lo laboral para mitigar los posibles conflictos de interés que pudiesen aparecer al momento de tomar decisiones. Para ello es prescindible que las empresas cuenten con políticas y procedimientos claros para que cada accionista sepa manejarse al interior de la organización.

La planificación estratégica, en la cual toda empresa sin importar su tamaño tiene que afianzar su proceso direccionamiento estratégico, mismo que consiste en definir a donde se requiere llegar a través del tiempo (visión) la razón de ser (misión), los valores y los objetivos del negocio. Definido el rumbo del negocio, se diseñarán los planes de acción, de manera que se fortalezcan los factores básicos de competencia de la empresa sin descuidar el esquema de seguimiento y evaluación que puede ser plasmado en un BSC (Balance Scorecard). El BSC facilita a las empresas familiares y a la pyme en general, la integración del día a día (corto plazo) con los planes futuros (largo plazo), y lo prepara para afrontar de mejor manera el reto de ser rentables y crecer, en otras palabras, las prepara para ser negocios con una gestión empresarial eficiente. La planificación estratégica ayuda a las empresas identificarse a sí mismas, partiendo de las premisas: que tengo(FODA), que o quien soy(misión), hacia donde voy(visión), como lo hago (BSC y gestión por procesos); esto implica adoptar una cultura de cambio, tanto en lo empresarial como personal, ya que las organizaciones exitosas basan su gestión en la identificación y generación de ventajas competitivas apalancadas en el profesionalismo de sus colaboradores, la adopción de tecnología y la perspicacia de sus timoneles. Las empresas familiares se han encontrado a sí mismas compitiendo en un entorno más turbulento impulsado por la nueva tecnología, valores sociales pluralistas, intensificación de la competencia, una economía global y políticas y regulaciones cambiantes. La planificación en la empresa familiar se suele centrar en: la planificación de la herencia y la sucesión. Estas metas resultan demasiado limitadas para la empresa familiar de hoy. Los autores presentan el nuevo concepto del proceso de planificación paralela y explican la forma de integrar las necesidades y expectativas de los sistemas familiares y empresariales a fin de crear una unidad organiza emprendedora los miembros de la familia pueden ser una enorme fortaleza o una debilidad potencial para la empresa familiar. Si se les ignora sean cuales sean sus planes, inevitablemente se debilita la empresa. Las empresas familiares que enfatizan en exceso el sistema empresarial y consiguiente disminuyen la atención del sistema familiar. Con frecuencia dan lugar a familias en las que sus miembros no se relacionan entre ellos y psicológicamente compiten con la empresa. Con el desarrollo de un plan de continuidad de empresa familiar se garantiza que los intereses de la familia se toman en consideración al tiempo que los de la empresa. Hay muchas ventajas en un proceso de planificación para la familia:-Anima a que la próxima generación y los familiares políticos aprendan de la historia y los 
valores de la familia--Refuerza un buen proceso de comunicación familiar.Respalda el desarrollo de los acuerdos familiares sobre asuntos tales como el empleo o la propiedad antes de que se tome una decisión especifica.-ofrece un proceso justo para la planificación y la toma de decisiones de la familia- Aclara las expectativas en torno a temas inherente difíciles como dinero, carreras profesionales y control. La planificación es un elemento esencial para la supervivencia y el éxito de una organización. En la empresa familiar, planificar la estrategia de negocio entraña conjugar simultáneamente tres aspectos: gestión de la empresa, propiedad y familia. Estas tres dimensiones deben desarrollarse de modo conjunto y evolucionar en paralelo en el tiempo, si perder de vista que las decisiones estratégicas conviven con sensibilidades, lazos familiares y afectos personales. Para ser eficaces, las decisiones deben ser plasmadas, recogidas y vehiculadas jurídicamente de una forma adecuada, de manera que la planificación legal desempeña un papel fundamental en la planificación estratégica. Este es precisamente el mensaje que transmite la presente obra: la planificación legal como elemento determinante del éxito o fracaso de una empresa familiar. A través de la exposición de historias y casos reales, Frederrick D. Lipman nos ofrece una amena e interesante herramienta de reflexión y aprendizaje buenas y malas prácticas. La sucesión es una de las decisiones más duras que se deben tomar en una empresa familiar, pero es también una de las más importantes: una sucesión bien estructurada puede preservar el negocio para futuras generaciones, mientras que una mal estructurada solo acabara generando caros litigios entre los miembros de la siguiente generación, y la venta definitiva de la empresa. La planificación de la sucesión suele diferir de manera notable de acuerdo a si es para una primera generación, con un solo propietario del negocio, o para una segunda generación de hermanos socios o una tercera formada por un consorcio de primos. la planificación de la sucesión tiene que ser parte de un plan estratégico para la empresa. El proceso de preparación de un plan estratégico de negocio que incluya el asunto de la sucesión ayuda a reflexionar sobre el futuro. La comunicación fluida entre los miembros de la familia es importante para mantener la armonía, y se convierte en una válvula para airear las quejas que plantea la planificación de la sucesión. La sucesión es la transmisión del mando y de la propiedad de una generación a otra. Por tanto, tiene dos vertientes: una en la gerencia y otra en la propiedad. Ambas son fundamentales y están estrechamente interrelacionadas la planificación anticipada y en vida de la secesión es crítica. No designar ni preparar con tiempo a los sucesores suele dejar a los herederos ante serios problemas que, por 1 general, imponen una fuerte presión emocional. Desde el punto de vista de la propiedad, la legislación varía de país a país y es un tema jurídico. Hay que pensar a la sucesión como proceso y no como hecho puntual. Es decir, no como algo que ocurre en un instante, sino como el resultado de todo un trabajo de largo aliento. Las empresas familiares suelen ser mucho mejor evaluadas por el mercado cuando pasan esta transición sin ningún conflicto ni problema que altere de manera importante sus proyectos y flujos futuros. Planificar su propia sucesión y 
retirarse a tiempo de la gestión ejecutiva para dar paso a la siguiente generación o a un gerente no familiar es uno de los actos de mayor valentía que pueda realizar un empresario en su vida

En base a lo analizado a las empresas familiares en lo que respecto a la planificación se pudo observar que tanto las pequeñas como medianas empresas deben realizar una planificación estratégica que sirva de guía para los dueños y también para poder tomar decisiones en beneficio de la empresa familiar ya que estas empresas tienen una característica importante que es la sucesión o 'próxima generación quienes serán los responsables del futuro de la empresa de allí la importancia de tener una buena planificación para alcanzar los objetivos de la empresa.

Una de las principales debilidades que tienen las empresas familiares es la falta de planificación en la sucesión de la empresa familiar. Una de las causas de mortalidad de la empresa familiar no responde tanto a problemas económicos, de mercado. La principal causa tiene que ver con el reemplazo del dueño de la empresa o de los fundadores de la empresa, con la desaparición del líder familiar y la falta de planificación para la sucesión generacional. Otro aspecto muy importante dentro de planificación familiar es definir primero la visión, misión, valores y objetivos estratégicos, así como un análisis para construir la estrategia de la empresa familiar.

Prevenir y planificar adecuadamente la sucesión de la empresa tiene consecuencias positivas para la supervivencia de las empresas familiares.

\section{Fortaleza y debilidades de las Famipymes.}

> Según (Ginebra, 1997), (Villanueva, 2015) , (Alejandro Macias, 2015), (Alejandro Macias, 2015), (Ing. Mirian Cevallos Sornoza, 2008)En uno y otro lugar de este trabajo, hemos tratado de contribuir a una clarificación que se nos hace imprescindible: la que permia diferenciar los fenómenos realmente propios y específicos de as empresas familiares de aquellos que un análisis más detenido hace ver como muy generales. El nepotismo como hemos señalado hay factores en la familia que presionan a la dirección de la empresa a favorecer a los parientes en detrimento de la mejor elección de personas, o a pagar más uno por ser de la familia. Aunque conviene señalar que esto último se da mayormente en $3^{\mathrm{a}}$ y cuarta generación. El endeudamiento: La existencia de feudos dentro de las organizaciones es un hecho muy general: departamentos enteros que se hacen impermeables a la acción de los demás En esta ocasión comentaremos sobre cuatro factores que impactan de manera positiva el desempeño de las empresas familiares, y a la vez, limitan su desarrollo si no se manejan adecuadamente. Es decir, las fortalezas de las empresas familiares se pueden convertir en debilidades, todo es cuestión de cómo se están manejando. Podemos ver o imaginar estos factores como las cuatro patas de una mesa, si alguno de ellos nos falla entonces la mesa se caerá, por muy bien que estén las demás patas. El primer factor de fortaleza es su visión de largo plazo, en las empresas familiares el objetivo general es la permanencia durante mucho tiempo en el mercado, incluso, la idea del 
fundador, en la mayoría de los casos, es que su empresa continúe con sus hijos y sus nietos. Esta visión de largo plazo quita la presión de los directores generales de las empresas no familiares, que tienen como criterios de su evaluación, la generación de utilidades en el corto plazo, por lo tanto, aunque tengan proyectos muy atractivos para consolidar la posición competitiva de su empresa, si tienen retornos sobre la inversión a largo plazo, no los toman en cuenta. Quedarse en su puesto está en función de darles a los accionistas resultados de rentabilidad en el corto plazo. Sin embargo, el riesgo de caer en excesos en esta visión a largo plazo, es comprometer la operación de la empresa en el corto o mediano plazo, es importante tener un equilibrio con el desarrollo de proyectos rentables de corto plazo que nos permitan obtener fondos para la innovación en proyectos de largo alcance. Un segundo factor de fortaleza son las relaciones de afecto, en la medida en que tenemos una relación cimentada en el cariño, se favorece la comunicación, la confianza, se agiliza la toma de decisiones, se tienen procesos menos burocráticos, se mejora la actitud de servicio tanto entre los empleados como con los clientes, todos estos son ingredientes muy necesarios para lograr la competitividad de las empresas. Es indiscutible que las organizaciones funcionan mejor cuando tenemos relaciones afectuosas que cuando tenemos relaciones hostiles entre los colaboradores. En la empresa familiar es posible tener un mejor clima organizacional que en las no familiares, el cariño por nuestros familiares nos lleva a disfrutar del reconocimiento y logros de los miembros de la familia, es más fácil aceptar el éxito de un familiar que de otras personas. Por otra parte, estas relaciones de afecto, con frecuencia nos llevan a tomar decisiones de contratar a familiares sin las competencias necesarias para realizar un trabajo, o bien, para mantener en una posición a un familiar que no se desempeña en forma adecuada, siendo paternalistas en nuestras decisiones. Los ascensos o promociones de puestos que consideran únicamente los lazos de sangre hacen muy difícil lograr el éxito en las empresas familiares. El tercer factor de fortaleza es el sentimiento de propiedad, sabernos dueños de la empresa familiar implica un mayor compromiso, una mayor dedicación, todo lo que hacemos será en nuestro beneficio, no se trabaja para el bien de "otros". De igual forma que cuando vivimos en una casa propia o en una casa rentada, en nuestra casa sabemos que todo nuestro esfuerzo por cuidar o mejorar la sala, la cocina, las recámaras, etc., será en nuestro beneficio y no para el dueño de la vivienda. En muchas ocasiones, la propiedad está relacionada con el apellido de la familia, esto promueve que las organizaciones tengan políticas de responsabilidad social corporativa, en beneficio de los empleados, el medio ambiente y la sociedad en general, cuidando la empresa y el prestigio de la familia de no caer en actividades de corrupción o no éticas.

Sin embargo, al sentirnos "dueños", podemos caer en el error del autoritarismo, en pensar, la empresa es mía y "hago con ella lo que me dé la gana", ejerciendo un estilo de liderazgo basado en el poder, sin tomar en cuenta las opiniones de los demás, esto lo que provoca es la falta de participación del personal, este tipo de 
ambiente no favorece la colaboración, y por consecuencia, será difícil también llegar a la institucionalización o profesionalización de la empresa que garantice la permanencia del negocio en el futuro. El cuarto factor de fortaleza es la estabilidad del personal, se dice que la rotación de personal es la peor huelga de una empresa, porque perdemos talento y potencial de gente que se ha capacitado en la organización, gente que conoce y tiene experiencia en el ramo que estamos trabajando. En las empresas familiares el personal tiene mayor lealtad a la organización, se sienten con mayor seguridad en el trabajo, esto los hace más productivos y se tienen mayores resultados.

Si el personal está contento, satisfecho y seguro en el trabajo, como habíamos comentado, además de tener mayor productividad, permitirá mayor continuidad en los planes de la empresa, incluso, para los proveedores, clientes o distribuidores habrá un mayor nivel de certeza en las operaciones con la empresa. Por otra parte, el riesgo que podemos caer en este factor, es que los miembros de la familia por esta "seguridad", confían en que no serán despedidos independientemente de su efectividad en el trabajo, esto influirá en un bajo desempeño de la empresa. Además, en casos extremos, la estabilidad nos puede llevar a no contratar profesionales con las competencias necesarias para afrontar los nuevos retos del entorno actual de los negocios. No hay organizaciones perfectas donde no haya algo que se pueda hacer mejor.

En resumen, los cuatro factores relevantes o las cuatro patas de la mesa en las empresas familiares son: 1. Visión de largo plazo o Descuidar operación del día a día, dejando las cosas para después.2. Relaciones de afecto o Nepotismo.

3. Propiedad y compromiso) o Autoritarismo donde "sólo mis chicharrones truenan".4. Estabilidad y seguridad o Conformismo. Las familias deciden emprender juntas o se van integrando en torno a la empresa por diversas razones, una muy importante es precisamente una de sus principales fortalezas: la confianza que se pueden tener entre ellos. Lograr confianza entre los miembros de una empresa puede ser el resultado de mucho tiempo, mucho trabajo y dinero, y aun así no se tiene garantizado mientras que en muchas familias ya es un activo importante, que muchas veces va acompañado además de un fuerte líder que no es cuestionado. De modo que se puede iniciar una relación de negocios con mucha ventaja. Sin embargo, esa misma confianza puede convertirse en la debilidad de la empresa si las reglas que aplican a los empleados y/o socios no familiares no se aplican del mismo modo a los familiares. Exigiendo capacidad y resultados para ocupar un puesto o percibir un sueldo. A veces resultará mejor "becar" a algún familiar con un ingreso obtenido de las utilidades del negocio antes que darle una responsabilidad para la que no tiene la actitud ni la capacidad de llevar a cabo, ya que no solo afecta los resultados del negocio por su trabajo sino también por el efecto desmotivador para el resto del equipo. Otra regla importante es mantener una sana separación entre los asuntos familiares y los de del negocio, así por ejemplo la llamada de atención que debe realizar el líder a uno de los familiares porque su desempeño podría estar fallando, no debe 
afectar la relación familiar y no debe reflejarse por lo tanto en las reuniones familiares. Estas reglas también aplican en empresas con miembros amigos de muchos años que podría tener relaciones casi familiares. Las empresas familiares se crean y tiene éxito en todos los países y como el resto de las empresas deben seguir un proceso de desarrollo para institucionalizarse. Por otro lado, los problemas por lo que pueda atravesar un negocio, por ejemplo, falta de ventas o de ingresos, afectan directamente a toda la familia inmediatamente, de modo que se debe ser suficientemente inteligente para manejarlo afectando lo menos posible la relación de la empresa. Una herramienta muy poderosa que debe aprender a manejar el líder es el manejo de los conflictos; ayudando a manejarlo a cada uno de los miembros a través del perdón, que cada vez es una técnica más extendida en las empresas. Eso requiere que el líder gestione el proceso y provoque por tanto un rápido y adecuado perdón entre los miembros. Prueba cada estrategia y mide los resultados, para que puedas decidir si esa estrategia está funcionando o debes eliminarla cando antes. Y recuerda que, aunque puedes encontrar referencias del mercado en cuanto margen de utilidad, enfócate en mejorar el tuyo continuamente y tu negocio seguro prosperará. Lansberg (1988) destaca cómo la mayoría de las empresas familiares prefieren desarrollar sus negocios nacionales a acometer nuevas oportunidades de crecimiento internacional. Así, Nieto (2003) demuestra empíricamente, que las pymes familiares parecen mostrar una orientación preferente hacia mercados locales y poseen un ámbito geográfico más reducido que el resto de PYMES. Sin embargo, los resultados obtenidos por Fernández (2004) señalan como las empresas cuya estructura accionarial pertenece Fuentes Lombardo, G.; Vallejo Martos, M. C.; Fernández Ortiz, R. Aspectos determinantes en la internacionalización de la empresa familiar - básicamente a una familia tienen una mayor presencia en los mercados exteriores vía exportación que las empresas no familiares. Además, Gallo y Sveen (1991) y Gudmundson et al. (1999) nos aportan que las empresas familiares, por sus propias características, a menudo desarrollan estrategias limitadas a cubrir las necesidades de sus clientes en los mercados locales donde actúan y tienen sistemas de información y control poco desarrollados. Por ello, afirman que son más reacias a expandirse internacionalmente, ya que las estrategias que desarrollan podrían ser difíciles de cambiar debido a que el sistema familiar influye sobre el negocio.

Fortalezas. - El conocimiento y La experiencia en el negocio. - Un gran conocimiento del negocio por la dedicación de toda una vida al mismo (miembros de generaciones actuales y anteriores).

Visión a largo plazo. - Existe el deseo de que la empresa se transmita a la siguiente generación pese a no estar clara la continuidad del negocio por la existencia de un alto grado de incertidumbre propia y del entorno. Compromiso, dedicación y orgullo familiar. - Gran esfuerzo y sacrificio de los familiares por la empresa, entrega y disponibilidad total. Orgullo de los familiares por el negocio, 
el deseo de querer hacer las cosas bien, querer desarrollarlas, espíritu emprendedor e inquietud.

Lealtad, confianza y comunicación. - Los trabajadores de la empresa llevan muchos años trabajando en la compañía, existe buena comunicación debido a la cercanía de estas personas a la dirección y propiedad de la empresa.

Satisfacción del cliente y preocupación por la calidad. - La calidad de los productos y el servicio al cliente se convierten en los objetivos prioritarios ante la imposibilidad de competir por volumen, variedad de productos y/o precio.

Autonomía en la forma de actuar y financiera. - Su independencia para tomar decisiones les permite dar respuesta de mayor calidad a los clientes. Empresas poco endeudadas que intentan sobrevivir con los recursos que generan, acudiendo a financiación externa solo en casos extremos. Los propietarios en ocasiones no viven de la empresa, sino que siguen siendo accionistas por el cariño que sienten por el negocio y/o por el status social que genera ser dueño de una bodega

Debilidades. - La sucesión o relevo generacional. -1 Sucesión no planificada, es un tema que suele quedar siempre pospuesto.

Nepotismo. - Falta de profesionalización motivada porque los puestos directivos de la empresa están ocupados en su mayoría por personas de la familia sin la suficiente formación. Familiares ocupando puestos directivos sin la adecuada formación, lo que genera conflictos con directivos externos que deben asumir estos huecos en determinados puestos de dirección de la empresa. Conflictos entre los intereses de directivos familiares y directivos ajenos a la familia.

Discordias familiares que influyen en el negocio. - Estas discordias vienen motivadas por los distintos intereses y visiones del negocio que tienen familiares de distintas generaciones. Nuevos miembros de la familia desean incorporarse al negocio sin la aceptación de los directivos familiares actuales

Falta de una clara definición estructural y solapamiento de roles. - Las empresas tienen estructuras organizativas inadecuadas para operar en el extranjero, sin departamentos, funciones, responsabilidades y sin personal especializado. Problemas de información interna para satisfacer la demanda de clientes extranjeros. Falta de especialización del personal.

Problemas de financiación. - La familia opta por la reinversión de los beneficios que genera la empresa, pero no acude a financiación externa por lo que no se dispone de capital suficiente para desarrollar nuevos proyectos de inversión.

Estilo de dirección autocrático y paternalista. - El estilo de dirección es autocrático, toma de decisiones muy centralizada en una persona. Participar en el accionario o la gestión de una empresa de la propia familia puede proporcionar enormes satisfacciones; en una empresa familiar es más fácil darse cuenta de que la persona debe ser valorada más allá de los resultados que produce, y de que está administrando un legado que hay que transmitir a la siguiente generación. Todo ello lleva a un mayor compromiso con el proyecto empresarial común, basado en una motivación más elevada que la puramente económica, que permite superar los paradigmas habituales en estrategia corporativa. Participar en una empresa 
familiar puede proporcionar grandes satisfacciones, mayores y más elevadas que las económicas.

\section{Principales fortalezas.}

Compromiso y dedicación. El hecho de que los puestos relevantes de dirección de la empresa estén ocupados frecuentemente por miembros de la familia, así como la identificación del prestigio de la marca con el prestigio familiar, motiva que la entrega de sus miembros al negocio sea permanente e ilimitada.

Unidad y cohesión del equipo directivo. Las relaciones de proximidad existentes entre los miembros de un grupo familiar que ocupan cargos directivos pueden facilitar, en mayor medida, el conocimiento y comprensión de las decisiones adoptadas por los otros familiares gestores.

Expectativas de inversión a largo plazo. En este tipo de organizaciones el futuro económico de la familia suele estar ligado, de una manera importante al éxito y continuidad del negocio a largo plazo, lo que contribuye a su estabilidad.

Conocimiento profundo del producto y del mercado "objetivo". La mayor parte de las empresas familiares suelen dedicarse a la fabricación o distribución de productos o servicios de alta calidad muy específicos, con origen en el ingenio y la visión emprendedora del fundador.

\section{Principales debilidades.}

La gran mayoría de los problemas específicos que deben afrontar las empresas familiares tienen su origen, precisamente, en la estrecha relación existente entre el negocio y la familia. Esta vinculación puede acabar generando una confusión entre las necesidades empresariales y las necesidades familiares, lo que resulta especialmente crítico en el momento de la sucesión. De este modo, los principales problemas a los que se debe enfrentar la empresa familiar podrían sintetizarse en los siguientes:

Trasvase de los conflictos familiares a la empresa y viceversa. No existe separación entre el plano familiar y el plano empresarial, de modo que existe un alto riesgo de que las desavenencias familiares afecten a las decisiones empresariales, que acabarán estando dirigidas por motivaciones personales. 20 Del mismo modo, resulta frecuente que los problemas del negocio se discutan en el ámbito familiar, distorsionando las relaciones personales entre sus miembros. Confusión entre propiedad, trabajo y capacidad de dirección. Resulta habitual en las empresas familiares el considerar que los miembros de la familia poseen, en virtud de dicha condición, el derecho y el deber de trabajar en el negocio, con independencia de su voluntad, preparación y capacidad profesional. No existe, en estos casos, una regulación del acceso y salida de familiares en la empresa ni una definición clara de sus funciones, de manera que se colocan en puestos de responsabilidad a personas no cualificadas. Además existe una gran reticencia a la incorporación de profesionales ajenos al entorno familiar en puestos directivos. Incoherencias retributivas entre empleados familiares y no familiares. En conexión con lo señalado en el punto anterior, en muchos supuestos de negocios 
de carácter familiar la retribución satisfecha a los trabajadores-familiares no guarda correspondencia alguna con sus capacidades y méritos reales, tanto por exceso como por defecto

Una empresa familiar se considera cuando gran parte de la propiedad le corresponde y es administrada por la familia. La contribución de la empresa familiar a la estabilidad económica de un país es una de sus características importantes. La actividad de las empresas familiares viene creando en la sociedad un inmenso impacto económico y, con su actividad, contribuyen a la creación de nuevos productos y también a brindar servicios que representan la mayor parte de la demanda en el mercado.

Un aspecto muy importante de este tipo de empresas es que los familiares se encuentran comprometidos con la forma de una atención al cliente de una manera más cordial y esmerada y una calidad de servicio más alta, lo que produce un mayor grado de confianza y notoriedad entre los clientes. El fundador de la empresa juega un papel fundamental en la vida de las empresas familiares ya que siente un mayor grado de responsabilidad con sus trabajadores, lo que se traslada en una buena estimación de estos hacia él y en sentirse que forman parte de la familia. Influye también en las empresas familiares la toma de decisiones empresariales entre propietarios y directivos ya que pueden darse ciertas discrepancias entre los mismos.

\section{Estadísticas.}

Seguin (IDE BUSSINESS SCHOOL, 2007)

\begin{tabular}{|c|c|c|}
\hline \multicolumn{3}{|c|}{ Principales datos de las empresas del Ecuador } \\
\hline Número de empresas & Familiares & No Familiares \\
\hline $\begin{array}{l}\text { \% sobre total de empresas Grandes } \\
\text { \% sobre total de PYMES } \\
\text { \% sobre total de Microempresas } \\
\text { \% sobre total empresas (estimado) }\end{array}$ & $\begin{array}{r}77 \% \\
90 \% \\
100 \% \\
89 \%\end{array}$ & $\begin{array}{r}23 \% \\
10 \% \\
0 \% \\
11 \%\end{array}$ \\
\hline \multicolumn{3}{|l|}{ Empleo } \\
\hline $\begin{array}{l}\text { \# de empleos formales estimados (millones) } \\
\text { \% Empleo formal }\end{array}$ & $\begin{array}{r}16 \\
93 \%\end{array}$ & 0,12 \\
\hline \multicolumn{3}{|l|}{ Rentabilidad } \\
\hline $\begin{array}{l}\text { Rent. Neta } \\
\text { ROE } \\
\text { ROA }\end{array}$ & $\begin{array}{l}3 \% \\
13 \% \\
5 \%\end{array}$ & $\begin{array}{r}8 \% \\
23 \% \\
9 \%\end{array}$ \\
\hline \multicolumn{3}{|l|}{ Ventas } \\
\hline $\begin{array}{l}\text { Variación ventas (prom } 5 \text { años) } \\
\text { Variación ventas (ultimo año) } \\
\text { \% respecto al PIB (solo empresas grandes) } \\
\% \text { respecto a las ventas de } 500 \text { empresas más } \\
\text { grandes ( } 2005 \text { ) }\end{array}$ & $\begin{array}{l}25 \% \\
22 \% \\
51 \% \\
65 \%\end{array}$ & $\begin{array}{l}25 \% \\
31 \% \\
27 \% \\
35 \%\end{array}$ \\
\hline $\begin{array}{l}\text { \% respecto a las ventas de } 500 \text { empresas más } \\
\text { grandes ( } 1996 \text { ) }\end{array}$ & $75 \%$ & $25 \%$ \\
\hline Fuente: IDE - Dpta de Investigación (En base a estimaciones) & & \\
\hline
\end{tabular}

Según la tabla se puede decir que el ecuador el 100\% de las microempresas son familiares, además el $90 \%$ de las pymes también pertenecen a familias, las empresas familiares ofrecen un $93 \%$ de empleo a las personas del Ecuador es decir que en las fami pymes 
ISSN 2602-8506

Vol. 1 / No.1/ pág. 5-31. Enero-Marzo/2017

www.visionariodigital.org

están empleadas la mayoría de personas por ende dichas empresas son las que tienen más rentabilidad en el país y las que contribuyen al país.

Según (Instituto Nacional de Estadística yCensos)

Ventas totales 2013 (dólares) Pequeñas, medianas y grandes

2.978.459.585

empresas

Ventas Nacionales 2013

2.885 .570 .245

Exportaciones 2013

92.889 .340

Ventas 2013 por actividad económica (dólares) Pequeñas,

2.978.459.585 medianas y grandes empresas

Agricultura, ganadería, silvicultura y pesca

112.105.987

Explotación minas y canteras

Industrias manufactureras

686.237 .521

Suministro electricidad, gas, vapor y aire acondicionado

51.358 .687

Distribución agua; alcantarillado, desechos y saneamiento

586.100

Construcción

50.525 .546

Comercio, reparación automotores y motocicletas

1.613 .803 .054

Transporte y almacenamiento

36.024 .143

Actividades de alojamiento y de servicio de comidas

28.931.316

Información y comunicación

9.472 .445

Actividades financieras y de seguros

119.105 .222

Actividades inmobiliarias

7.704 .920

Actividades profesionales, científicas y técnicas

87.727 .487

Actividades de servicios administrativos y de apoyo

Administración pública y defensa, seguridad social

Enseñanza

70.551 .725

Actividades de atención a la salud humana y asistencia social

16.166 .166

Artes, entretenimiento y recreación

Otras actividades de servicios

38.409 .602

En el siguiente cuadro podemos conocer que las pequeñas, medianas y grandes empresas en Ecuador en el año 2013 han efectuado ventas que han favorecido a distintos sectores que tiene el Ecuador, podemos agregar que por la influencia de empresas familiares o las famipymes hemos incrementado como país más de lo que se pudo haber esperado la magnitud que tuvo las ventas es una cantidad acorde a lo que se podía estimar y valorar a las empresas sobre todo sobreponiendo que cada sector contribuye con las necesidades del país y ante todo valiéndose de manera extraordinaria de las empresas familiares que aporta con nuevas expectativas de rumbo.

Las empresas familiares ocupan un lugar muy esencial en el país ya que por algunos años han estado más tiempo y se han abierto al país de manera exuberante en tal parte que sus ganancias nos ayudan al país en seguir emprendiendo más empresas familiares. 
Las ventas que se efectúan en las empresas familiares podrían contribuir de alguna manera con el bienestar de las personas lo que si nos ayudan es a dar más fuentes de trabajo en el país y dar más soluciones de vida a distintas familias que hay.

Con todo esto el emprender una empresa familiar es favorable para la sociedad ya que impulsa a crear más productos nuevos que contribuyen con la superación del país.

\section{Análisis general.}

Según los resultados de la encuesta realizada hacia los empresarios familiares y los empresarios de las famipymes en la provincia de Tungurahua se evidencio que de las 200 encuestas hechas que es el $100 \%$ el $25 \%$ nos comentó que son dueños que se pusieron las empresas por satisfacer las necesidades que tenían de acuerdo a la situación que está generando en el país, el otro $25 \%$ nos aduce que por su magnitud de personas se han tenido que poner sus empresas en el centro de Ambato ya que esto proporciona más ingresos económicos a la misma, lo que no sucede con otros cantones aledaños a Ambato que por su poca magnitud de gente no se puede generar muchos ingresos.

El $25 \%$ de las encuestas nos comentan que han tenido que generar empresas y familiares por lo que han tenido que asociarse con familiares y así colocar la empresa y ayudarse entre sí para poder sobrellevar la situación económica de la misma.

Y finalmente el otro $25 \%$ de las encuestas nos dicen que debería haber capacitaciones de acuerdo al sector en el que están posicionándose en el mercado; para que así puedan saber que necesidades no más tiene las personas que de acuerdo a sus gustos, preferencias y muchas cosas que necesita saber el gerente o dueño de la empresa. Con estos resultados se puede concluir que las empresas familiares son aquellas que han salido de acuerdo a la necesidad de cada familia, ya que es más importante tener algo de uno al no tener nada.

\section{Conclusiones.}

- La famipymes contribuyen a la sociedad con más fuentes de trabajo y sobre todo a tener una economía más estable con precios que ayuden y satisfagan a la personas.

- Las empresas familiares son aquellas que cubren necesidades de las familias que necesitan surgir e independizarse de un trabajo que no les llena sus necesidades.

- Se concluye que a su vez las empresas familiares y las famipymes son aquellas que facilitan la vida en las familias ya que la misma familia es aquella que aporta con ideas para que se abra en el campo de la vida empresaria.

- Las empresas familiares deben tener confiabilidad por parte de sus miembros, y a la vez deben comprometerse a solucionar cada uno de los problemas que se les presente en el transcurso del desarrollo de la entidad y a brindar un producto o servicio que tenga el apellido familiar, el cual le dará mayor realce a la empresa. 
- Las ventajas que tienen las famipymes es que existen miembros de la familia que tienen el conocimiento en los negocios, la capacidad para tomar las mejores daciones a largo plazo y cuentan con dinero y tiempo para poder crear y al mismo tiempo crecer en el mercado.

- Las empresas familiares aportan de gran manera con la economía de un país ya que no solo aporta con sus productos y servicios sino también genera oportunidades de empleo.

- Las empresas familiares también tienen sus fortalezas y oportunidades, siendo una fortaleza de gran importancia el compromiso que tienen sus dueños con la empresa para motivar a sus colaboradores, una debilidad en común es la de los conflictos familiares que afectan negativamente a la empresa ya que los problemas son traslados a la interna de la empresa

\section{BIBLIOGRAFÍA}

Bueno, S. V. (28 de Febrero de 2014). UniversoPymes. Recuperado el 27 de Junio de 2015, de UniversoPymes: http://universopyme.mx/?p=3742

Casani, F., Llorente, A., \& Pérez, E. (2009). Economía de la empresa $2^{\circ}$ Bachillerato. Editex.

Cleri , C. (2007). El libro de las PYMES. Argentina: Granica.

De Aguilar, E. (1998). Beneficios fiscales en la empresa familiar: patrimonio y sucesiones. España: Caja de Ahorros Y Pensiones de Barceclona.

Ginebra, J. (2005). Las empresas familiares: su dirección y su continuidad. México: PANORAMA .

Goméz Betancourt, G. (2006). ¿Son iguales todas las empresas familiares?: caminos por recorrer. Colombia: Norma.

Hambra, J. (07 de Agosto de 2013). Buenos negocios. Recuperado el 27 de Junio de 2015, de Buenos negocios: http://www.buenosnegocios.com/notas/486-parientes-laempresa-ventajas-y-desventajas

Martínez Echezárraga, J. (2011). Empresas familiares. Reto al destino: Claves para perdurar con éxito. Argentina: Granica.

Mucc, O. O. (2008). Empresas familiares: Funcionamiento e identidad. Argentina : EUDEM.

Negocios, E. d. (01 de Julio de 2014). eaeprogramas. Recuperado el 27 de Junio de 2015, de eaeprogramas: http://www.eaeprogramas.es/empresa-familiar/2014/07/quees-una-empresa-familiar-concepto-y-caracteristicas.htm

Regalado, R. (2006). Las Mipymes en Latinoamerica. Argentina. 
Ronqullo, J. L. (2006). Administracion basica de la empresa familiar. México: PANORAMA.

Ruiz, A. G. (Julio-Septiembre de 2011). espae.espol.edu.ec. Recuperado el 27 de Junio de 2015, de espae.espol.edu.ec: http://www.espae.espol.edu.ec/images/documentos/publicaciones/publicaciones _medios/empresasfamiliares.pdf

Urbano, D., \& Toledano , N. (2008). Invitación al emprendimiento: Una aproximación a la creación de empresas. España: UOC.

Alejandro Macias. (2015). Coachparaempresas. Recuperado el 28 de Junio de 2015, de Coachparaempresas: http://www.coachparaempresas.com/empresas-familiaresuna-debilidad-o-una-fortaleza/

Alexandra, F. (2013). Reposito Espe. Recuperado el 28 de Junio de 2015, de Reposito Espe: http://repositorio.espe.edu.ec/bitstream/21000/7756/1/AC-PDE-ESPE047459.pdf

Echezárraga, J. M. (2010). Empresas familiares: Reto al destino. Argentina: Granica S.A. Frederick. (2011). Guía para las empresas familiares. España: P.A.P.F.

Ginebra, J. (1997). Las empresas familiares: su dirección y su continuidad. México: Panorama.

Ing. Mirian Cevallos Sornoza. (2008). RepositorioIAEN. Recuperado el 28 de Junio de 2015 de

RepositorioIAEN: http://repositorio.iaen.edu.ec/bitstream/24000/404/1/IAEN-M035-2008

Mucci, O. O. (2008). Empresas familiares: Funcionamiento e identidad. Argentina: Eudem.

Vallejo Martos. Manuel Carlos. (2007). Obtenido de file://D:/Documentos\%20de\%20Xtratech/Downloads/390-1364-1-PB.pdf

Villanueva, J. E. (2015). ITESM. Recuperado el 2015 de Junio de 2015, de ITESM: http://www.itesm.mx/wps/wcm/connect/snc/portal\%20informativo/opinion\%20 y\%20analisis/firmas/dr.\%20jorge\%20eduardo\%20gomez\%20villanueva/op(30e ne14)jorgegomez

Ward, R. S. (2003). La planificación estratégica de la familia empresaria: Barcelona: Deusto.

Instituto Nacional de Estadística yCensos. (s.f.). Instituto Nacional de Estadística y Censos. Obtenido de http://aplicaciones2.ecuadorencifras.gob.ec/dashboard2/pagina1.php

IDE BUSSINESS SCHOOL. (Abril de 2007). Recuperado el 05 de Julio de 2015, de IDE BUSSINESS SCHOOL: http://investiga.ide.edu.ec/index.php/revista-abril2007/680-todo-queda-en-familia

Basco, R. (2006). LA INVESTIGACIÓN EN LA EMPRESA FAMILIAR: “UN DEBATE SOBRE LA EXISTENCIA DE UN CAMPO INDEPENDIENTE". Investigaciones Europeas de Dirección y Economía de la Empresa , pp.33-54.

Salazar , G. (10 de Mayo de 2005). Degerencia.com. Obtenido de http://www.degerencia.com/articulos.php?artid=733 
Flores, A. (2013). Repositorio.espe.edu.ec. Recuperado el 06 de Agosto de 2015, de Repositorio.espe.edu.ec:

http://repositorio.espe.edu.ec/bitstream/21000/7756/1/AC-PDE-ESPE047459.pdf

\section{Para citar el artículo indexado.}

Bonilla H., Moncayo A. \& Solís L. (2017). Las FAMIPYMES como un modelo de desarrollo en la economía de Tungurahua.Revista electrónica Visionario Digital 1(1), 0531.

\section{Ciencia LDigital}

El artículo que se publica es de exclusiva responsabilidad de los autores y no necesariamente reflejan el pensamiento de la Revista Ciencia Digital.

El articulo queda en propiedad de la revista y, por tanto, su publicación parcial y/o total en otro medio tiene que ser autorizado por el director de la Revista Ciencia Digital 